\title{
Systematic biases and uncertainties of Hipparcos parallax
}

\author{
X. P. Pan \\ Jet Propulsion Laboratory, California Institute of Technology, Pasadena,CA 91007, USA \\ email: Xiaopei.Pan@jpl.nasa.gov
}

\begin{abstract}
Ground-based optical/IR interferometers have provided strong support to the spacebased astrometric mission Hipparcos ever since the Hipparcos instrument was in operation in 1989. Interferometric observations also produced critical corrections of orbital motion to many targets, including radio stars, which link the Hipparcos system to the International Celestial Reference Frame (ICRF). In particular, orbital parallax from interferometers confirmed the $10 \%$ bias of the Pleiades distance from Hipparcos, and thus avoids revision of classical astronomy. Significant offsets and errors of Hipparcos parallax introduced by binary jitters are demonstrated in this work. By comparing the Hipparcos results with long baseline interferometry and other techniques including spectroscopy, multi-color photometry, Main-Sequence fitting, light curve measurements, Lunar occultation, Fine Guidance Sensor, etc., systematic biases and uncertainties of Hipparcos parallaxes are investigated and analyzed. We have established good models for major error sources of Hipparcos parallax, such as zonal bias, binary jitters, and luminositydependent errors. The lessons learned from the systematic biases of Hipparcos parallax are valuable to future space missions like SIM and Gaia.
\end{abstract}

Keywords. astrometry, techniques: interferometric, methods: data analysis

\section{Introduction}

Classical astrometry has limited accuracy and precision mainly for two reasons, i.e. the effects of diffraction and turbulence of the atmosphere. The Hipparcos spacecraft was launched into space to avoid atmospheric perturbations and achieve a milli-arcsecond (mas) precision. Ground-based optical/IR interferometers use long baselines $\left(5 * 10^{7} \lambda\right)$ to solve the difficulties of diffraction limits, and obtain unprecedented angular resolution of milli-arcsec. In addition, interferometers can freeze the atmosphere within seeing limits to obtain sub-mas measurement precision. Since 1987 the Mark III interferometer on Mt. Wilson has routinely provided scientific results in astrometry with high resolution and high accuracy (Shao, M. et al. 1988). For the first time in astronomy, spectroscopic binaries of milli-arcsec separation with large magnitude differences have been resolved with sub-mas precision (Pan et al. 1990). Combined with spectroscopic observations, the Mark III Interferometer provides orbital parallax with milliarcsec precision. A good example is the distance to the Hyades cluster, which was determined accurately via a binary star $\theta^{2}$ Tau as $22.9 \pm 0.9$ mas in 1992 (Pan, Shao \& Colavita 1992a), $22.0 \pm 1.2$ mas in 1995 (Tomkin, Pan \& McCarthy 1995) and 21.0 00.8 mas in 1997 (Torres, Stefanik \& Latham 1997). The errors in the above distance are limited by radial velocity measurements, not by interferometry. In 1997 Hyades distance was confirmed by Hipparcos measurements as $21.89 \pm 0.83$ mas for that star (Perryman 1997) and $21.58 \pm 0.13$ mas for the Hyades cluster (Brown et al. 1997). In particular the radio star, Algol (HR 936), which is the prototype of eclipsing binary and a triple system, had its outer orbit determined (Pan, Shao \& Colavita 1993). The orbit of Algol from the Mark III interferometer provided accurate corrections to link the Hipparcos reference frame to the International Celestial Reference 
Frame(ICRF). Besides, the Mark III interferometer determined wide-angle star positions with ten mas precision in 1989 (Shao, M., Colavita, M. M, et al. 1990). Those wide-angle star positions provided critical references to confirm the high precision capabilities of Hipparcos preliminary measurements when the Hipparcos mission was troubled by its undesired orbit in 1989. The internal working note by the Hipparcos team admired valuable and speedy contributions from the Mark III interferometer (Perryman et al. 1990). Since the Hipparcos results are deeply troubled by binary stars, many accurate orbits of binary stars, which can be resolved only by interferometers, have been used in the Hipparcos catalogue in order to reduce the effects of binarity jitter in Hipparcos parallax (Pan et al. 1992b). Ever since the Hipparcos catalogue was published in 1997, the arguments on systematic errors in the Pleiades distance from Hipparcos have never stopped. Using ten years of observations from both the Mark III and the PTI interferometers the brightest binary star in the Pleiades cluster, Atlas, has its orbit determined without a help of the spectroscopic means, for the first time since development of interferometry (Pan, Shao \& Kulkarni 2004). The orbital distance of the Pleiades, which has the big advantages of being without the influence of interstellar extinction, confirmed the $10 \%$ systematic bias of Hipparcos data for the Pleiades.

\section{Comparisons between interferometry and Hipparcos}

Binary stars not only play fundamental roles in astronomy and astrophysics, and also are extremely useful to check astronomical instrument performance. Binary stars have different separation, different color, different magnitude in 4-dimensional observations, and can be used to study resolution, accuracy and precision conveniently. For example, the Hipparcos catalogue used the interferometric orbit of $\alpha$ And (Pan et al. 1992b) and obtained its photocentric semi-major axis $\left(a_{0}\right.$ ") to be $6.47 \pm 1.16$ mas (Perryman 1997). Recently a group of astronomers use Hipparcos and spectroscopic results to get the latest $a_{0}$ " of $\alpha$ And equal to $7.26 \pm 0.38$ mas (Jancart, Jorissen, Babusiaux \& Pourbaix). Those results, however, troubled them, even though the precision of 0.38 mas is much better than before. The problem is that value of $a_{0}$ " produces $\Delta m=3.9$ mag, which is inconsistent with the Mark III's measurements. The paper said that "The origin of this discrepancy is unknown". If the error analysis has not been understood properly, the small error bars cannot represent accurate measurements and precise analysis. Here we compare interferometric performance with Hipparcos measurements by calculating the same photocentric semi-major axis from two different techniques as shown in Table 1. It is obvious that interferometric precision $(0.1 \mathrm{mas})$ is more than an order of magnitude better than the Hipparcos $(\sigma=1-5$ mas). Some authors combined the Hipparcos results with spectroscopy, and made the offset of $\alpha$ And much worse than before because of limited precision and number of visits for that target. The average of systematic offsets between Hipparcos and interferometry is about 1.3 mas, which is consistent with general error estimate of Hipparcos. We notice that many binaries, such as $\theta^{2}$ Tau with $a_{0} "=$ 4 mas, cannot be resolved by Hipparcos. Also, the minimum resolution of Hipparcos measurements is about 2 mas. It is impossible for Hipparcos to detect extra-solar planets like hot Jupiters (signature of hot Jupiters is about 0.1 mas or less).

\section{Error models of Hipparcos parallax}

Many papers have concluded that errors of Hipparcos parallax have random Gaussian distribution. By using an average of 100 stars parallax, the mean parallax of that cluster can have an error of $1 / \sqrt{100}=0.1$ mas assuming 1 mas error for each star. That technique 
Table 1. Comparison of Photocentric Orbits between Interferometry and Hipparcos

\begin{tabular}{crcccrc}
\hline $\begin{array}{c}\text { star } \\
(\mathrm{HIP})\end{array}$ & $\begin{array}{c}\text { a" } \\
\text { (mas) }\end{array}$ & $\begin{array}{c}\text { mass } \\
\text { Ratio }\end{array}$ & $\begin{array}{c}\Delta m a g \\
(\mathrm{mag})\end{array}$ & $\begin{array}{c}c a l-a_{0} " \\
(\mathrm{mas})\end{array}$ & \multicolumn{1}{c}{$\begin{array}{c}\text { Hip }-a_{0} " \\
\text { (mas) }\end{array}$} & $\begin{array}{c}\text { Offsets } \\
(\mathrm{mas})\end{array}$ \\
\hline$\alpha$ And & $24.15 \pm 0.13$ & 0.33 & 2.63 & 4.66 & $6.47 \pm 1.16$ & 1.81 \\
& & & & & $7.26 \pm 0.38$ & 2.60 \\
$\beta$ Ari & $36.10 \pm 0.30$ & 0.64 & 1.99 & 20.00 & $11.32 \pm 1.33$ & 8.68 \\
& & & & & $12.50 \pm 1.20$ & 7.50 \\
Atlas & $12.94 \pm 0.11$ & 0.42 & 1.68 & 3.15 & $4.23 \pm 0.97$ & 1.08 \\
HIP 91009 & $4.40 \pm 0.05$ & 0.47 & 2.50 & 1.66 & $3.11 \pm 0.97$ & 1.45 \\
HIP 109176 & $10.33 \pm 0.10$ & 0.38 & 1.70 & 2.16 & $4.07 \pm 0.27$ & 1.91 \\
HIP 101382 & $15.37 \pm 0.03$ & 0.44 & 2.40 & 5.25 & $5.24 \pm 0.66$ & 0.01 \\
HIP 23453 & $16.20 \pm 0.10$ & 0.45 & 2.20 & 5.48 & $4.18 \pm 0.90$ & 1.30 \\
HIP 24608 & $56.47 \pm 0.05$ & 0.49 & 0.15 & 1.25 & $2.16 \pm 0.60$ & 0.91 \\
HIP 96683 & $23.70 \pm 0.04$ & 0.49 & 0.30 & 1.39 & $1.60 \pm 0.61$ & 0.21 \\
HIP 28360 & $3.30 \pm 0.10$ & 0.49 & 0.20 & 0.12 & $-0.54 \pm 0.76$ & 0.66 \\
HIP 99473 & $3.20 \pm 0.10$ & 0.45 & 1.53 & 0.80 & $1.25 \pm 1.04$ & 0.45 \\
HIP 2912 & $6.69 \pm 0.05$ & 0.45 & 0.40 & 0.29 & $1.84 \pm 0.98$ & 1.55 \\
HIP 14576 & $94.61 \pm 0.22$ & 0.27 & 2.92 & 19.88 & $19.00 \pm 0.57$ & 0.88 \\
$\theta^{2}$ Tau & $18.80 \pm 0.06$ & 0.47 & 1.13 & 3.84 & & 3.84 \\
HIP 10280 & $2.10 \pm 0.90$ & 0.46 & 4.00 & 0.91 & & 0.91 \\
HIP 69226 & $3.39 \pm 0.05$ & 0.50 & 0.60 & 0.44 & & 0.44 \\
HIP 3810 & $6.52 \pm 0.06$ & 0.49 & 0.11 & 0.09 & & 0.09 \\
HIP 47508 & $4.46 \pm 0.01$ & 0.47 & 0.91 & 0.74 & & 0.74 \\
HIP 7564 & $4.94 \pm 0.02$ & 0.56 & 2.06 & 2.13 & & 2.13 \\
HIP 69974 & $19.76 \pm 0.08$ & 0.48 & 0.63 & 2.31 & & 2.31 \\
HIP 19762 & $2.78 \pm 0.06$ & 0.46 & 0.56 & 0.25 & & 0.25 \\
\hline
\end{tabular}

brought many contradictory results for many clusters, and RR Lyr stars. In fact many error sources, such as instrument errors, binary jitter, zonal errors produced significant systematic errors in Hipparcos parallax(Pan \& Makarov 2007). A typical example is the Hipparcos parallax of $23.41 \pm 2.44$ mas for HIP 32104 after correction of its orbital motion $\left(a_{0} "=8.83 \pm 1.85\right.$ mas) (Perryman 1997). We used accurate orbital parameters and obtained a new parallax as $20.56 \pm 0.71$ mas, which demonstrates a binary jitter of 2.3 mas $(>3 \sigma)$. We plot Hipparcos parallax error distributions as shown in Figure 1 for all 120000 stars, where the errors can be described as

$$
\sigma_{H i p}^{2}=\sigma_{\text {sys }}^{2} / f_{N}+\tau(\operatorname{mag}) / f_{N}
$$

In the above model $\sigma_{s y s}$ is the systematic error; $\tau(\mathrm{mag})$ is the brightness dependent error; $f_{N}$ is the number of visits. It is interesting to notice that the systematic error of 0.77 mas is the dominant error source for the majority of stars $\left(f_{N}=25\right)$. When the stars are fainter than $8.7 \mathrm{mag}$, the brightness dependent errors are equal and larger than the systematic error. The obvious gap between 0.5 and 0.75 mas for bright stars $(<7$ mag) is strong evidence for the effects of number of visits. Since it is impossible to have less than 10 visits for a meaningful fit, many stars above the green line ("+") with errors of $\tilde{1} .5$ mas are meaningless.

\section{Conclusions}

Long baseline optical/IR interferometers provide orbit determinations with precisions at a sub-mas level, which is critical to correct for binary jitter in Hipparcos observations. It has been demonstrated that the threshold of orbit detectability for Hipparcos is comparable to a single measurement precision of a few mas only. In addition, more 


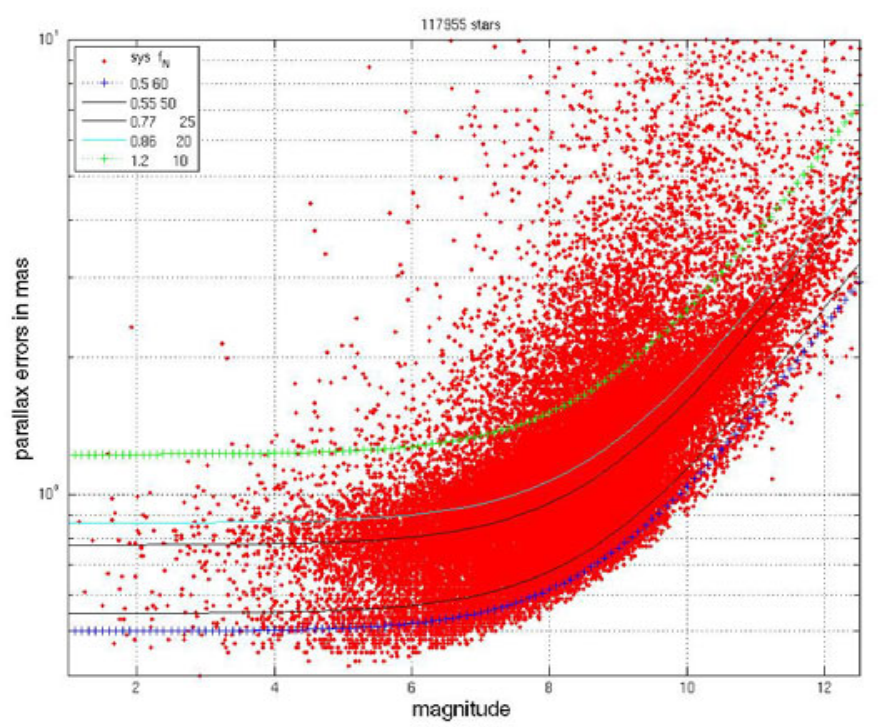

Figure 1. Error distributions of Hipparcos parallaxes

than $40 \%$ of binaries cannot be detected by Hipparcos. It is impossible for Hipparcos to detect the signature of exoplanets. A new model of Hipparcos parallax demonstrates a term of systematic errors at 0.8 mas. That model provides useful tools for identifying objects with large biases. The most important lessons we learned from the Hipparcos mission is that we have to do deeper investigations on systematic biases by using various techniques. Future space missions like Gaia and SIM will face the same challenging tasks but at the level of $\mu a s$. It is important to use both Gaia and SIM to check and support each other.

\section{References}

Brown, A. G. A., Perryman, M. A. C., Kovalevsky, J., Robichon, N., Turon, C., \& Mermilliod, J.-C. 1997, Proceedings of the Hipparcos Venice;97 symposium, ESA SP-402 000, 681

Jancart, S., Jorissen, A., Babusiaux, C., \& Pourbaix, D. 2002, A\&A, 442, 365

Pan, Xiaopei, Shao, M. \& Colavita, M. M., Mozurkewich, D., Simon, R. S. and Johnston, K. J. 1990, ApJ 356, 641

Pan, Xiaopei, Shao, M., \& Colavita, M. M. 1992, in: H. McAlister \& W. Hartkopf (eds.), IAU Coll. 135, A. S. P. Conf. ser. Vol. 32, p. 502

Pan, Xiaopei, Shao, M., Colovita, Armstrong, J. T., M. M., Mozurkewich, D., Vivekanand, M, Denison, C. S., Simon, R. S., \& Johnston, K. J. 1992, ApJ 384, 624

Pan, Xiaopei, Shao, M., \& Colavita, M. M. 1993, ApJ (Letters) 413, L129

Pan, Xiaopei, Shao, M., \& Kulkarni, S. R. 2004, Nature 427, 326

Pan, Xiaopei \& Makarov, V. 2007, BAAS 39, No.1, 12

Perryman, M. A. C. 1990, Hipparcos Internal Working Note: Hipparcos/MK III report, 12/06

Perryman, M. A. C. 1997, The Hipparcos and Tycho Catologues, ESA SP-1200

Shao, M., Colavita, M. M., Hines, B. E., Staelin, D. H., Hutter, D. J., Johnston, K. J., Mozurkewich, D., Simon, R. S., \& Hershey, J. L., etc. 1988, A\& A 193, 357

Shao, M., Colavita, M. M, Hines, B. E., Hershey, J. L., Hughes, J. A., Hutter, D. J., Kaplan, G. H., Johnston, K. J., Mozurkewich, D., Simon, R. S., \& Pan, X. P. 1990, AJ 100, 1701

Tomkin, J., Pan, Xiaopei and McCarthy, K. 1995, AJ 109, 780

Torres, G., Stefanik, R. \& Latham, D. W. 1997, ApJ, 485, 167 